\title{
A farnesyltransferase inhibitor prevents both the onset and late progression of cardiovascular disease in a progeria mouse model
}

\author{
Brian C. Capell*†, Michelle Olive*, Michael R. Erdos*, Kan Cao*, Dina A. Faddah*, Urraca L. Tavarez*, \\ Karen N. Conneely ${ }^{\ddagger}$, Xuan Qu*, Hong San*, Santhi K. Ganesh*, Xiaoyan Chen*, Hedwig Avallone ${ }^{\S}$, \\ Frank D. Kolodgie§, Renu Virmani§, Elizabeth G. Nabel*ף, and Francis S. Collins*\| \\ *Genome Technology Branch, National Human Genome Research Institute, National Institutes of Health, Bethesda, MD 20892-8004; ${ }^{N}$ New York University \\ School of Medicine, New York, NY 10016; 'Department of Human Genetics, Emory University School of Medicine, Atlanta, GA 30322; ${ }^{\S} \mathrm{CVPath}$, \\ Gaithersburg, MD 20878; and "INational Heart, Lung, and Blood Institute, National Institutes of Health, Bethesda, MD 20892 \\ Contributed by Francis S. Collins, August 15, 2008 (sent for review July 15, 2008)
}

Hutchinson-Gilford progeria syndrome (HGPS) is the most dramatic form of human premature aging. Death occurs at a mean age of 13 years, usually from heart attack or stroke. Almost all cases of HGPS are caused by a de novo point mutation in the lamin A (LMNA) gene that results in production of a mutant lamin A protein termed progerin. This protein is permanently modified by a lipid farnesyl group, and acts as a dominant negative, disrupting nuclear structure. Treatment with farnesyltransferase inhibitors (FTIs) has been shown to prevent and even reverse this nuclear abnormality in cultured HGPS fibroblasts. We have previously created a mouse model of HGPS that shows progressive loss of vascular smooth muscle cells in the media of the large arteries, in a pattern that is strikingly similar to the cardiovascular disease seen in patients with HGPS. Here we show that the dose-dependent administration of the FTI tipifarnib (R115777, Zarnestra) to this HGPS mouse model can significantly prevent both the onset of the cardiovascular phenotype as well as the late progression of existing cardiovascular disease. These observations provide encouraging evidence for the current clinical trial of FTIs for this rare and devastating disease.

aging | Hutchinson-Gilford progeria syndrome | lamin | atherosclerosis | translation

$\mathbf{H}$ utchinson-Gilford progeria syndrome (HGPS) is characterized by a multitude of features reminiscent of normal aging. Most devastating, however, is the rapidly progressive arteriosclerotic disease that leads to death in most patients from cardiovascular complications during the early teen years (1). HGPS is caused by a mutation in the $L M N A$ gene (G608G), which encodes components of the nuclear lamina, a dynamic multi-component scaffolding network lying just beneath the inner nuclear membrane (2-4). This mutation activates a cryptic splice donor in exon 11 that produces progerin, which, as a result of a 50-aa internal deletion, cannot undergo the final proteolytic processing step in the lamin A maturation pathway and thus is left permanently modified by a lipid farnesyl group $(2,5,6)$. Transgenic mice carrying a copy of the human G608G LMNA mutation display a phenotype characterized by a dramatic and progressive loss of vascular smooth muscle cells (VSMCs) in the media of large arteries (7), a cardiovascular phenotype that is remarkably similar to the cardiovascular disease observed in autopsy studies of patients with $\operatorname{HGPS}(8,9)$.

The lamin A precursor, prelamin A, contains a C-terminal CAAX motif that is a signal for posttranslational farnesylation by the farnesyltransferase enzyme $(6,10)$. This is thought to localize the prelamin A to the nuclear membrane. Once there, the zinc metalloproteinase ZMPSTE24 cleaves off the final 15 aa at the $\mathrm{C}$ terminus, including the farnesyl group, allowing mature lamin A to be inserted into the nuclear lamina (11). In HGPS, this final cleavage site is deleted and progerin remains anchored in the nuclear membrane, disrupting the underlying lamina in a dominant negative fashion and leading to all of the downstream nuclear defects that are characteristic of HGPS, such as nuclear blebbing, heterochromatin disorganization, mislocalization of nuclear envelope proteins, and disrupted gene transcription (4, $5,12-15)$.

Several studies have demonstrated that suppressing this farnesylation step by pharmacologically inhibiting the farnesyltransferase enzyme leads to improvement in these cellular phenotypes, most notably the nuclear morphology of cultured HGPS skin fibroblasts (16-19). Furthermore, two progeroid mouse models have shown the ability of farnesyltransferase inhibitor (FTI) treatment to improve phenotypes $(20,21)$. However, these two models, one of which was a knockout of Zmpste24, and the other of which created a Lmna allele that could only produce progerin, did not include any cardiovascular phenotype and were not carried out using one of the two clinical candidate FTIs, tipifarnib (R115777, Zarnestra) or lonafarnib (SCH66336, Sarasar) (22). Given that occlusive cardiovascular disease is the cause of death in virtually all patients with HGPS, we sought to test the ability of the FTI R115777 to prevent the cardiovascular disease seen in transgenic G608G LMNA mice.

\section{Results}

R115777 mixed in transgenic mouse dough (a soft diet that allows for drug mixing and even distribution) or vehicle alone was orally administered at two doses, $150 \mathrm{mg} / \mathrm{kg} / \mathrm{day}$ and 450 $\mathrm{mg} / \mathrm{kg} /$ day, as these have been shown previously to be comparable to doses delivered to human patients. In vivo FTI activity was judged by Western blots of HDJ-2, a protein functionally unrelated to the lamins, but which also contains a CAAX motif that is farnesylated. In human trials, HDJ-2 is considered the standard biomarker of effective inhibition of farnesylation (23). Thirteen transgenic mice and 15 non-transgenic WT mice began treatment at weaning (i.e., age 1 month), and mice were euthanized and analyzed between 9 and 12 months of age. Full pathological examination revealed no consistent pathologic processes outside of the vascular system, and no apparent drug toxicity in WT or transgenic mice. Full serum chemistry panels revealed decreases in total cholesterol $(P=0.00032)$, total protein $(P=0.0027)$, and creatinine $(P=0.014)$, and increases

Author contributions: B.C.C., M.O., M.R.E., E.G.N., and F.S.C. designed research; B.C.C., M.O., M.R.E., K.C., D.A.F., U.L.T., X.Q., H.S., X.C., H.A., F.D.K., and R.V. performed research M.O. contributed new reagents/analytic tools; B.C.C., M.O., M.R.E., K.C., D.A.F., K.N.C., S.K.G., E.G.N., and F.S.C. analyzed data; and B.C.C., M.O., E.G.N., and F.S.C. wrote the paper

The authors declare no conflict of interest.

Freely available online through the PNAS open access option.

\|To whom correspondence should be addressed. E-mail: 4rancis@mail.nih.gov.

C 2008 by The National Academy of Sciences of the USA 

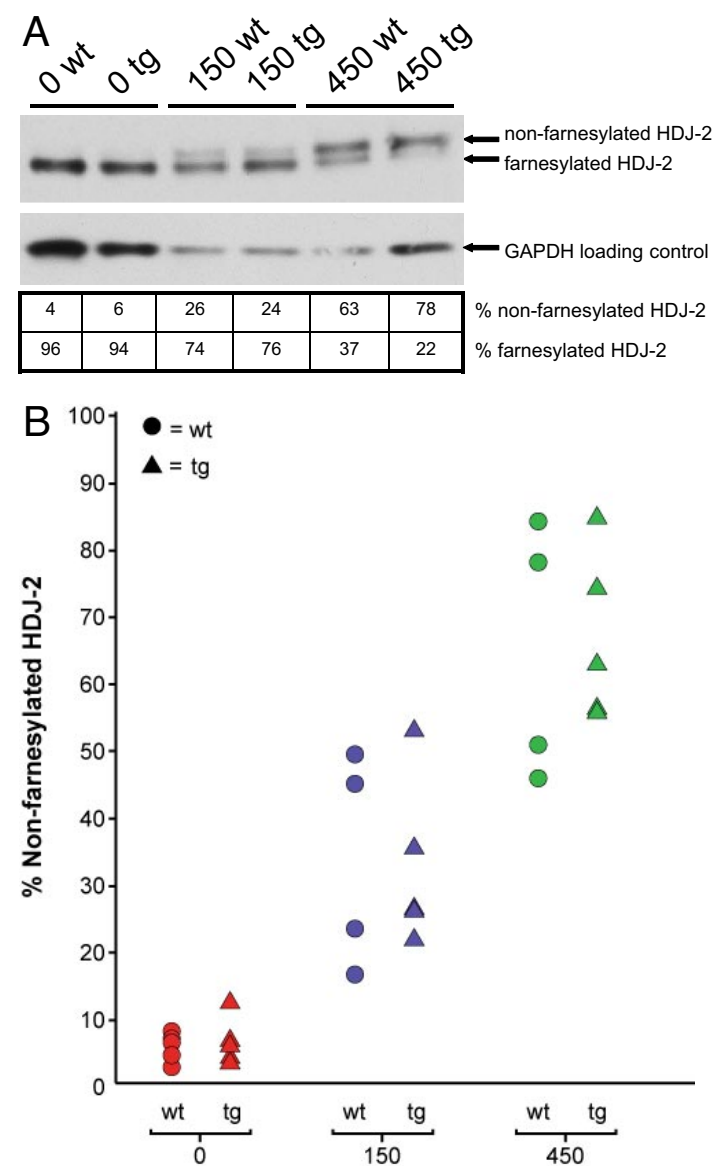

Fig. 1. Oral FTI treatment leads to increased levels of the biomarker nonfarnesylated HDJ-2. (A) Protein was extracted from mouse livers at the time of euthanasia. Western blotting of liver protein extracts demonstrates a doserelated increase in levels of non-farnesylated HDJ-2 (Upper) in comparison with farnesylated HDJ-2 (Lower) with FTI treatment. (B) All study mice are represented on a plot demonstrating the increasing levels of non-farnesylated HDJ-2 with increasing dose of FTI. Triangles represent transgenic G608G LMNA mice and circles represent non-transgenic WT controls.

in alkaline phosphatase $(P=0.016)$ for FTI-treated mice. Total body radiographs displayed no phenotype or effect of FTI treatment (data not shown).

Arterial sections, consisting of ascending aorta, descending aorta, and carotid artery, were analyzed using Movat pentachrome and hematoxylin/eosin stains, as well as immunofluorescence, and scored (as described in Methods) by 5 independent observers blinded to genotype and treatment group. Results for individual mice were plotted based upon the average pathology scores and compared with the amount of non-farnesylated HDJ-2 that appeared on Western blots of liver protein extracts obtained at the time of dissection. Non-farnesylated levels ranged from $2 \%$ to $12 \%$ in untreated mice, $16 \%$ to $53 \%$ in mice treated with $150 \mathrm{mg} / \mathrm{kg} /$ day of the FTI, and $45 \%$ to $85 \%$ in mice treated with $450 \mathrm{mg} / \mathrm{kg} /$ day (Fig. $1 A$ and $B$ ).

Blinded scoring of the immunofluorescence images (Fig. 2A) demonstrated significant improvement in both the ascending and descending aorta in terms of increases with FTI treatment in the abundance of VSMCs by smooth muscle $\alpha$-actin staining $(P=$ 0.0079 for descending aorta [Fig. 2B] and $P=0.016$ for ascending aorta [data not shown]) and by DAPI-positive nuclei in the medial layer of the vessels $(P=0.0014$ for descending aorta [Fig. 2B] and $P=0.0090$ for ascending aorta [data not shown]). This trend correlated both with dose and FTI biological effect as judged by the amount of non-farnesylated HDJ-2 (Fig. $2 B$ ). Strikingly, those mice with the highest levels of FTI effect (>70\% non-farnesylated HDJ-2) displayed the least pathology. Pathologic findings were not as extensive or consistent in the carotid arteries as in the aorta, and there was no trend with treatment in that site (data not shown).

Blinded scoring of Movat images also demonstrated improvement in VSMC integrity and a decrease in proteoglycan deposition in the descending aorta with FTI treatment. This trend correlated both with dose and FTI biological effect as judged by the amount of non-farnesylated HDJ-2 (Fig. $3 A$ and $B$ ). These improvement trends were significant upon removing from the analysis one untreated transgenic mouse that displayed no significant pathology (circled in Figs. $2 B$ and $3 B$ ), and appeared by subsequent quantitative RT-PCR to have silenced the transgene epigenetically (data not shown). In agreement with this finding, and unlike any other transgenic mouse in the study, this mouse did not show positive staining using the MAB3211 antibody, which detects only human lamin A and C (data not shown).

Encouraged by the ability of the FTI to prevent the cardiovascular phenotype when treatment is begun immediately at weaning, we next sought to examine the efficacy of R115777 in mice that are allowed time to develop disease. As children in the HGPS clinical trial have varying ages and disease states, whether FTIs are effective against preexisting disease is of key clinical importance. To this end, we allowed mice to reach the age of 9 months before beginning a 6-month course of oral FTI treatment. Given the greater efficacy of the $450 \mathrm{mg} / \mathrm{kg} /$ day dose in the initial study, 7 transgenic G608G LMNA mice and 9 WT control non-transgenic mice underwent treatment with either $450 \mathrm{mg} /$ $\mathrm{kg} /$ day or vehicle only. Following an identical analysis of the vasculature as described earlier, mice treated from 9 to 15 months of age displayed a highly significant arrest in the severe late progression of the loss of VSMCs seen in the untreated transgenic mice (Fig. $4 A$ and $B$ ). This halting of disease progression was dramatic and affected all vessels: descending aorta, ascending aorta, carotid artery, and abdominal aorta (Fig. $4 C)$. As many mice in this transgenic line already display significant disease at 9 months (Fig. $2 A$ and ref. 7), the nearly normal phenotype in the treated mice suggests that FTIs may also induce disease regression in mice that already manifest phenotypic changes.

\section{Discussion}

Given its extreme rarity (1 in 4 million live births), HGPS was long relegated to the role of a medical curiosity. With only the occasional case report appearing in the literature, its cause remained a mystery. However, upon the discovery that HGPS was almost always caused by a de novo single base change in the relatively well known $L M N A$ gene $(2,3)$, the field of HGPS research has rapidly expanded. The discovery that the mutant lamin A protein in HGPS (i.e., progerin) is permanently modified by a lipid farnesyl group, and that this appears to act as a dominant negative, pointed toward a possible therapeutic target. This target emerged as a particularly attractive one because of the existence of already well tested inhibitory compounds, the FTIs. Currently two such orally administered FTIs are in phase III clinical trials for cancer, motivated originally by the observation that the oncoprotein Ras is farnesylated. Although their efficacy in human clinical cancer trials has been somewhat disappointing, both FTIs have proven to be remarkably well tolerated (24).

Accumulating in vitro and in vivo data has indicated that FTIs may in fact offer some hope for patients with HGPS (16-21). However, these studies have not yet established whether FTIs might be efficacious in treating the most devastating aspect of the phenotype that leads to death in nearly all patients, cardio- 
A
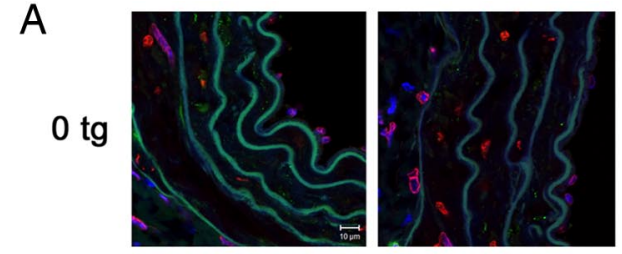

$150 \operatorname{tg}$
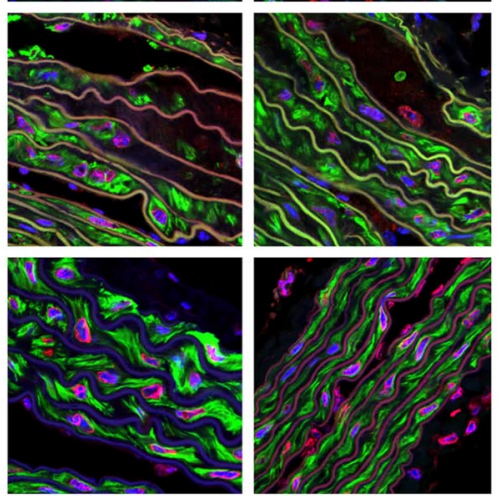

450 tg
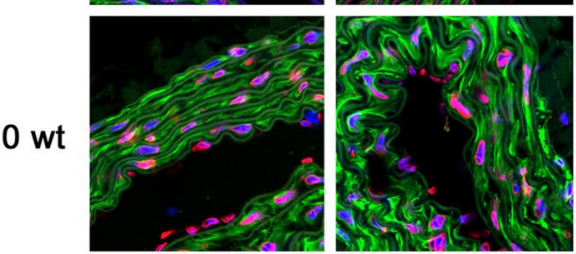

\section{B Descending Aorta VSMC Loss vs. FTI}
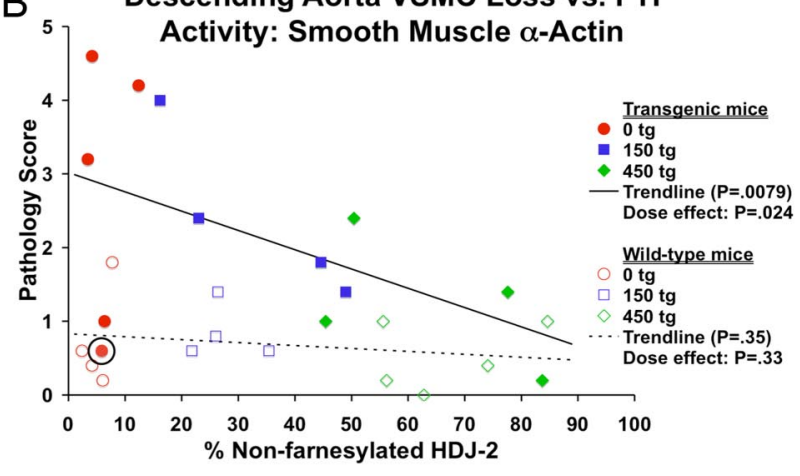

Descending Aorta VSMC Loss vs. FTI Activity: DAPI

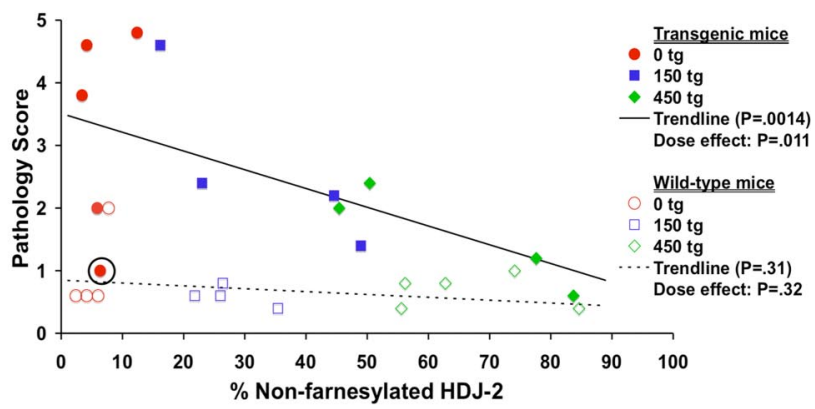

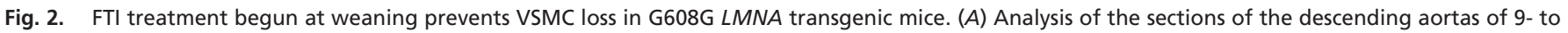

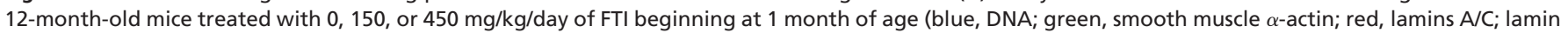

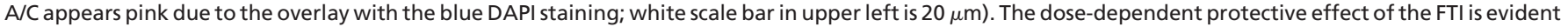

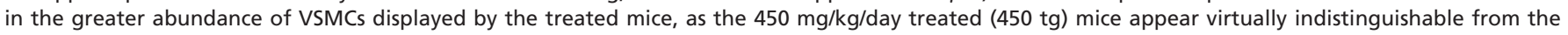

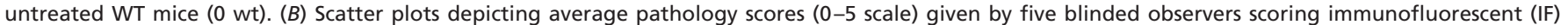

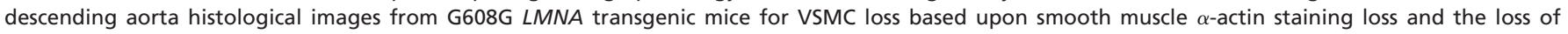

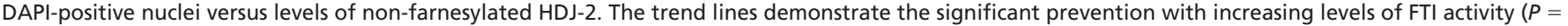

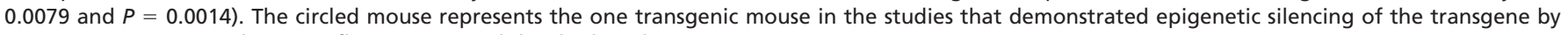
quantitative RT-PCR and immunofluorescence and thus had no disease.

vascular disease. Here, using a transgenic mouse model carrying the precise human HGPS mutation (LMNA G608G) and displaying a cardiovascular phenotype (a progressive loss of VSMCs in the medial layer of the large arteries) that notably recapitulates numerous aspects of the cardiovascular disease seen in HGPS (7, 8), we have shown that the FTI tipifarnib can successfully prevent both the appearance and the late progression of cardiovascular disease. Blinded scoring demonstrated highly significant improvements in VSMC loss and proteoglycan accumulation in mice that were begun on FTI treatment at weaning, and even in mice allowed to develop disease for 9 months before beginning a 6-month course of FTI treatment. The most significant improvement in the mice was seen in mice given the highest dose ( $450 \mathrm{mg} / \mathrm{kg} /$ day) and exhibiting the highest levels of FTI activity as judged by the biomarker nonfarnesylated HDJ-2.

Why the VSMCs are the most affected aspect of the vasculature in HGPS is not entirely clear $(7,8)$. Numerous theories have been proposed, including that these cells are especially vulnerable to the mechanical stress imposed on them in the large arteries, combined with the instability of the nuclear framework imposed by a defective lamina. The disrupted lamina, hypothesized to be disturbed in a dominant-negative fashion by the permanently farnesylated progerin protein anchoring itself to the overlying inner nuclear membrane, also leads to disorganization of the bound and underlying heterochromatin, as well as the numerous transcription factors bound to it $(4,8)$. These effects might be what is responsible for the greatly misregulated gene expression profiles seen in HGPS cells, particularly seen in genes involved in VSMC proliferation (15). Finally, it has recently been demonstrated that progerin can disrupt the proper differentiation of mesenchymal stem cells, thus potentially causing premature differentiation and exhaustion of this supply of cells that might otherwise assist in the regeneration of the damaged VSMCs (25). FTIs, by reducing the levels of farnesylated progerin that is available to act as this dominant negative, may decrease the ensuing nuclear damage caused by progerin as well as slow the mesenchymal stem cell defects that occur, thus allowing these cells to help repair areas of damaged vessels.

A clinical trial of the FTI lonafarnib was initiated in 2007 for 28 children with HGPS, although results are not expected for at least 1 year $(26,27)$. Notably, children with HGPS often display relatively stable cardiovascular disease for the majority of their lives before a dramatic acceleration in symptoms later in life (8). Thus, given the range of ages at enrollment of children in this trial (3-15 y), the ability of FTIs to have an effect on preexisting disease is a vital issue, as the children will undoubtedly display varying severities of cardiovascular disease. Whether the same ability to reverse disease seen in the mouse model can be reached in the children remains an important question. Likewise, the fragile state of children with the disease will necessitate close monitoring for unexpected toxicities.

Recently, another group has ameliorated the progeroid symptoms and extended the lifespan of Zmpste24-KO mice using the combined therapy of a statin and a bisphosphonate (28), two compounds that both act on the same mevalonate pathway on 
A

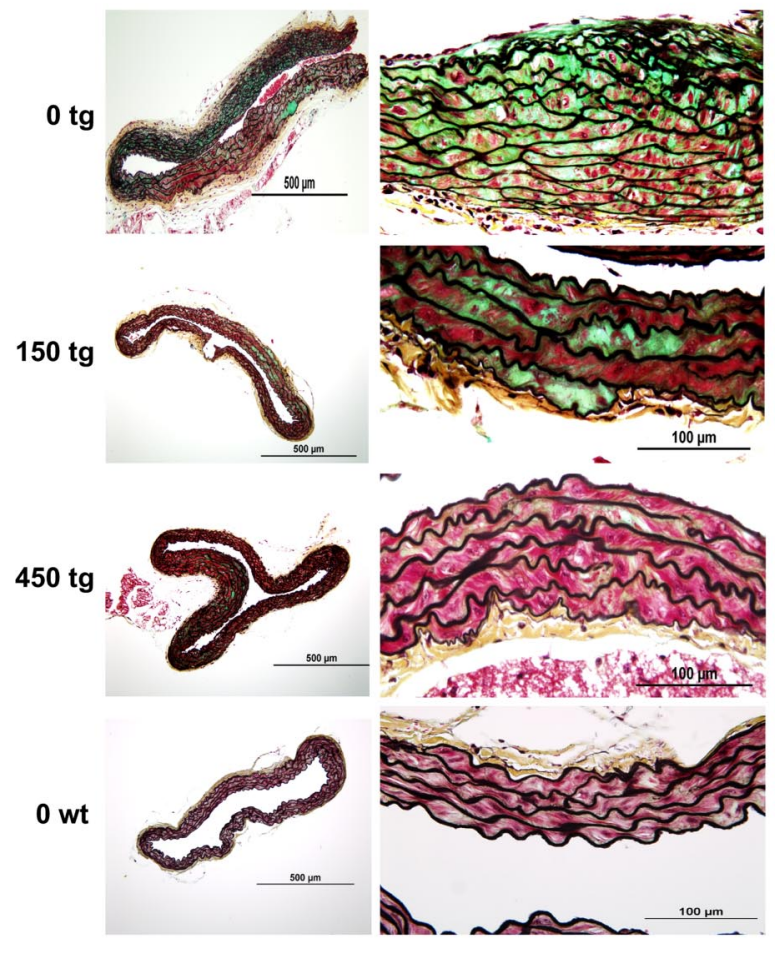

B

Descending Aorta VSMC Loss vs. FTI Activity: Movat

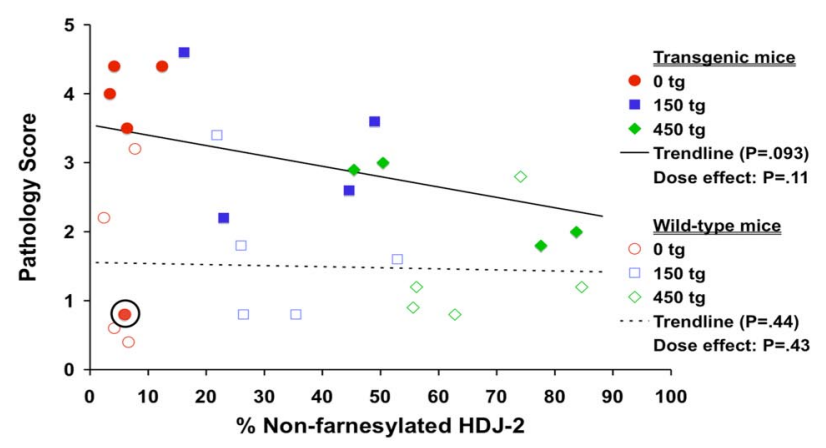

Descending Aorta Proteoglycan Deposition vs. FTI Activity: Movat

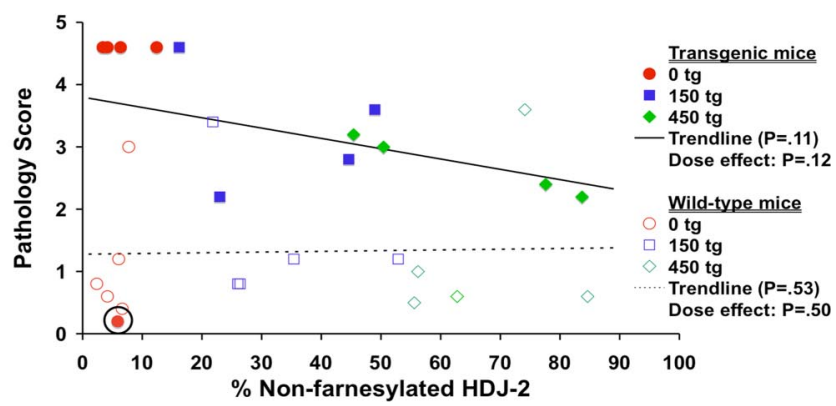

Fig. 3. FTI treatment begun at weaning prevents VSMC loss and proteoglycan accumulation in G608G LMNA transgenic mice. (A) Analysis of the sections of the Movat-stained descending aortas of 9- to 12-month-old mice treated with 0,150 , or $450 \mathrm{mg} / \mathrm{kg} / \mathrm{day}$ of FTI beginning at $1 \mathrm{month}$ of age (red, VSMCs; green, proteoglycan; black, elastin layers). The dose-dependent protective effect is clearly seen in the greater cellularity and reduced proteoglycan staining in the treated transgenic animals ( $150 \mathrm{tg}$ and $450 \mathrm{tg}$ ) in comparison with the untreated animals $(0 \mathrm{tg})$. (B) Scatter plots depicting average pathology scores $(0-5 \mathrm{scale})$ given by five blinded observers scoring the Movat descending aorta histological images from G608G LMNA transgenic mice for pathology in the forms of proteoglycan accumulation and VSMC loss versus levels of non-farnesylated HDJ-2. The circled mouse represents the one transgenic mouse in the studies that demonstrated epigenetic silencing of the transgene by quantitative RT-PCR and immunofluorescence and thus had no disease. The trend lines became significant upon removal of this outlier transgenic mouse from the analysis.

which FTIs act. However, as no direct comparison was made between this treatment and FTIs, the relative abilities of these drugs to act either alone or synergistically to treat HGPS remains an open question. And more broadly, the biology behind the role of prenylation in atherosclerosis in relation to the antiatherogenic effects of statins is only beginning to be explored (29). For example, FTIs have demonstrated the ability to prevent atherosclerosis in apolipoprotein E-deficient mice (30). Furthermore, with the accumulating recent evidence that all individuals produce small amounts of the mutant progerin protein, and that this may have some role in aging or longevity $(14,31,32)$, it is possible that the results presented here may have implications well beyond just this rare condition.

\section{Materials and Methods}

Mice. Generation, genotyping, and characterization of LMNA G608G mice was described previously (7). Mice were housed in a virus-free barrier facility with a 12-h light/dark cycle. Body weights were measured and recorded 3 times per week. Full pathological examination included the ascending aorta, descending aorta, carotid artery, abdominal aorta, external ear, femur, skin, liver, spleen, kidney, heart, skeletal muscle, testis, ovary, uterus, small intestine, large intestine, and esophagus. Full serum chemistry panels included glucose, total cholesterol, triglycerides, sodium, potassium, chloride, creatinine, BUN, albumin, calcium, magnesium, phosphorus, alkaline phosphatase, ALT, AST, amylase, creatine kinase, lactate dehydrogenase, total protein, and uric acid. All animals were genotyped again at the time of euthanasia, and for 5 mice (3 transgenic and 2 non-transgenic mice) in the group for which FTI treatment was delayed, there were discrepancies in the genotypes. Data from those animals were therefore removed from the analysis. Similarly, 2 mice (1 trans- genic and 1 non-transgenic) were removed from the analysis as a result of HDJ-2 Western blot results that were not interpretable. All animal use complied with the Animal Care and Use Committee guidelines under protocol G-03-05 (National Institutes of Health).

FTI. The FTI tipifarnib (R115777, Zarnestra) was mixed in transgenic mouse dough (Bio-Serv) at the doses of $150 \mathrm{mg} / \mathrm{kg} / \mathrm{day}$ or $450 \mathrm{mg} / \mathrm{kg} / \mathrm{day}$. Previous work showed that mixing the drug in the mouse diet allows maintenance of steady-state drug levels (C. Grubbs, personal communication). Each dose of R115777 was carefully manually kneaded into a kilogram of transgenic dough every 2 weeks. Five to 10 drops of food coloring were mixed into the dough during the mixing process to ensure that sufficient mixing of the drug throughout the dough had occurred. The vehicle control consisted of unmodified dough. Each mouse received a fresh 6-g ball of their specified transgenic dough each morning.

Western Blots. Total cellular protein extracts were isolated from homogenized mouse livers. Protein lysate was prepared in radioimmunoprecipitation assay buffer and included a mixture of proteinase inhibitors (Roche). A total of 10 $\mu \mathrm{g}$ of protein was loaded and electrophoresed on an $8 \%$ Tris-glycine mini gel (Invitrogen). Proteins were probed with mouse monoclonal [KA2A5.6] to HDJ-2 (Abcam 3089-500). Mouse monoclonal [6C5] to GAPDH (Abcam 8245) was used as a loading control. Percentages of farnesylated and nonfarnesylated HDJ-2 were examined using Photoshop version 7.0 (Adobe Systems) to determine the deviation of mean pixels across each band from the background.

Histochemistry. Tissues fixed in $2 \%$ paraformaldehyde and embedded in paraffin. Cross-sections were stained with hematoxylin/eosin and Movat pentachrome. Images were captured using an Olympus BX51 Microscope equipped with an Olympus DP11 digital camera. 
A

0 tg

$450 \mathrm{tg}$
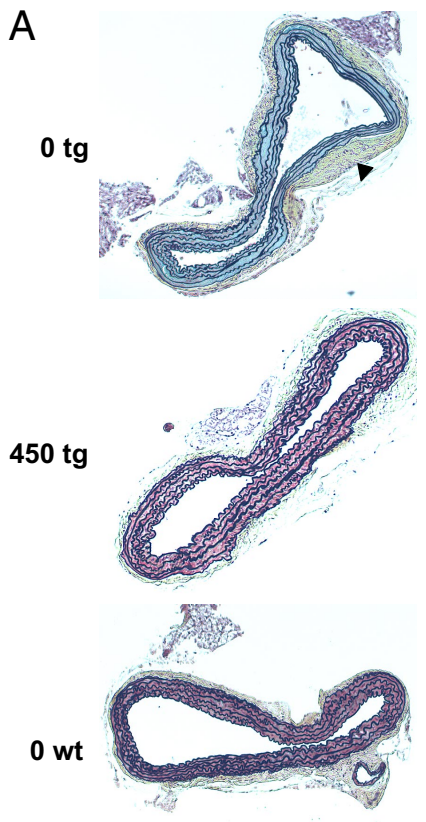

C

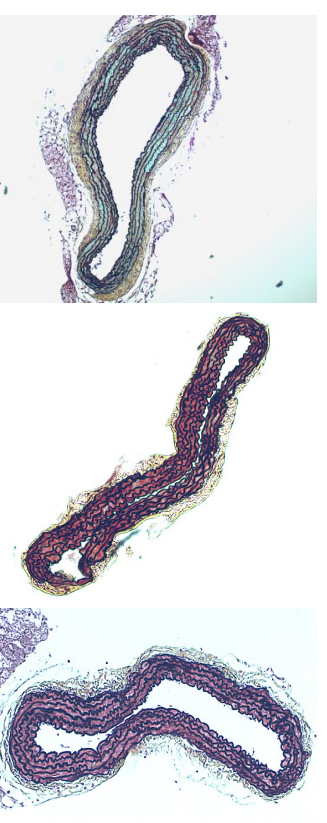

B
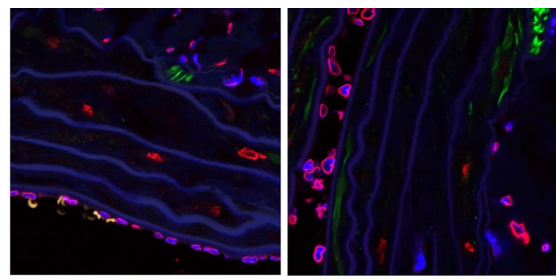

$450 \mathrm{tg}$
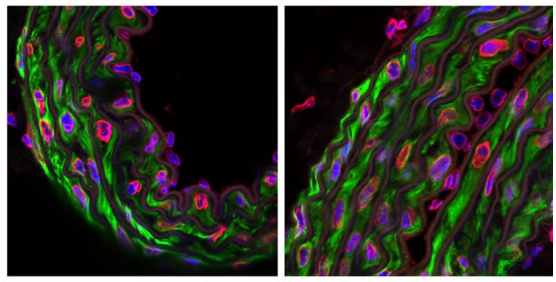

$0 \mathrm{wt}$
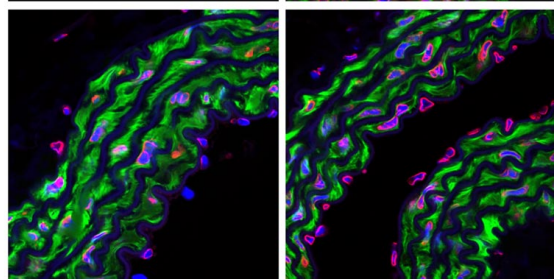

Descending Aorta VSMC Loss vs. FTI Activity: Movat
Ascending Aorta VSMC Loss vs. FTI Activity: Movat

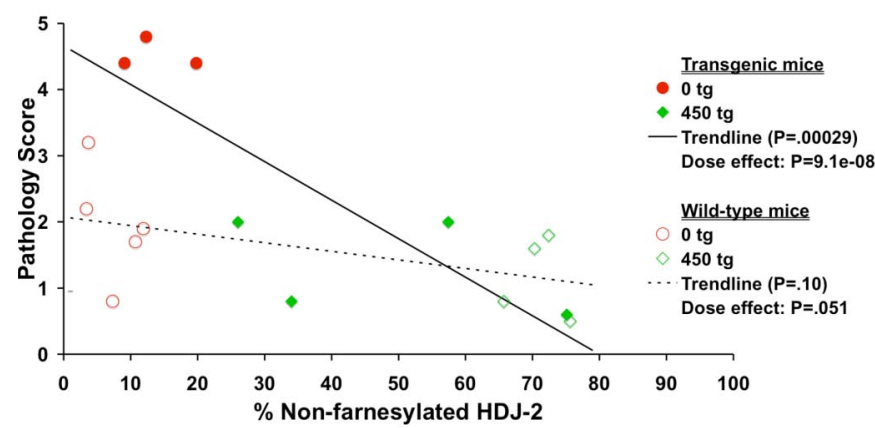

Carotid Artery Proteoglycan Deposition vs. FTI Activity: Movat

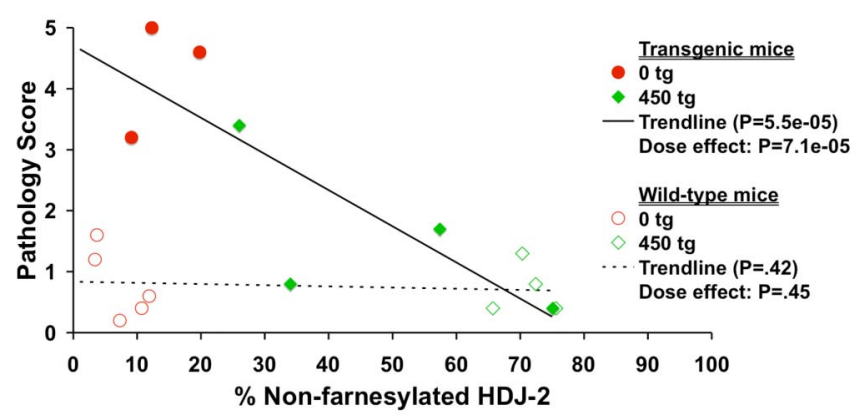

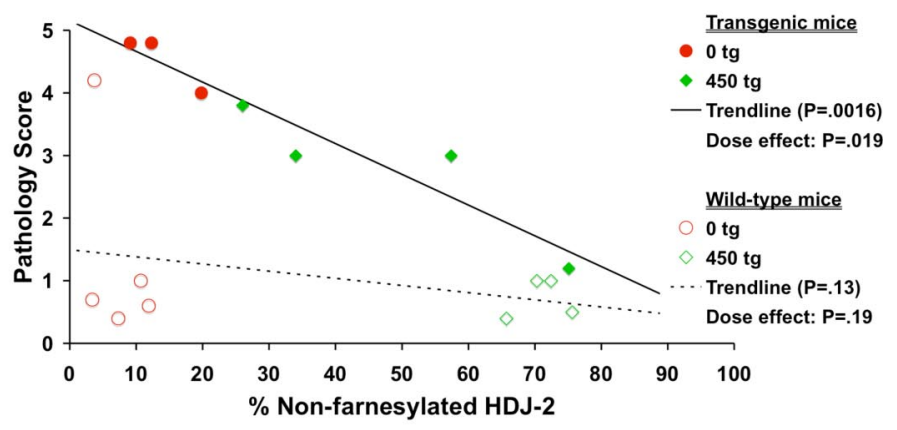

Abdominal Aorta Proteoglycan Deposition vs. FTI Activity: Movat

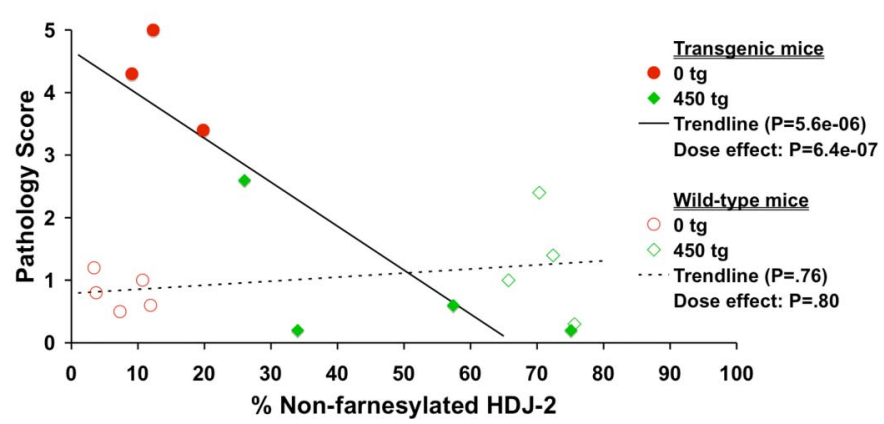

Fig. 4. FTI treatment begun at 9 months of age prevents the late progression of VSMC loss and proteoglycan accumulation in G608G LMNA transgenic mice. (A) Movat-stained sections of the descending aortas of four 15-month-old transgenic mice treated with 0 or $450 \mathrm{mg} / \mathrm{kg} / \mathrm{day}$ of FTl beginning at $9 \mathrm{months}$ of age (red, VSMCs; green, proteoglycan; black, elastin layers). Whereas untreated 15-month-old transgenic mice display a complete lack of VSMCs and abundant proteoglycan accumulation as well as marked adventitial thickening (black arrowhead), treated mice appear virtually identical to age-matched untreated WT controls. (B) Immunofluorescence of the sections of the descending aortas of four 15-month-old mice treated with 0 or $450 \mathrm{mg} / \mathrm{kg} / \mathrm{day}$ of FTI (blue, DNA; green, smooth muscle $\alpha$-actin; red, lamins A/C; lamin A/C appears pink due to the overlay with the blue DAPI staining; white scale bar in upper left is $20 \mu \mathrm{m}$ ). Both sets of sections demonstrate complete protection in mice beginning treatment at the late age of 9 months. Untreated vessels are almost completely devoid of any VSMCs in the media, and proteoglycan has accumulated to replace them. In contrast, FTI-treated mice appear quite similar to untreated WT control mice with the vessel media well populated by VSMCs. (C) A sampling of scatter plots depicting average pathology scores ( $0-5$ scale) given by five blinded observers scoring Movat descending aorta, ascending aorta, carotid artery, and abdominal aorta images from G608G LMNA transgenic mice treated from 9 to 15 months of age. The trend lines demonstrate the highly significant prevention trend with increasing levels of FTI activity in all plots. 
Immunohistochemistry. Staining for lamins was previously described (7). Antibody dilutions were: mouse monoclonal anti-lamin $A / C$ non-diluted (MAB3211; Chemicon); rabbit polyclonal anti-lamin A (1:10) (Ab2559; Abcam); monoclonal anti-smooth muscle $\alpha$-actin FITC-conjugated (1:100; clone 1A4; Sigma-Aldrich); Alexa Fluor 594-conjugated were used as secondary antibodies (1:1,000; Molecular Probes). Slides were mounted in DAPI-containing medium (Vector Laboratories). Fluorescence emission images were obtained with a confocal microscope system (LMS 510; Zeiss) and collected with $40 \times$ oil lenses.

Vessel Scoring Analysis. Five observers, blinded to both mouse genotype and FTI treatment group, scored both Movat pentachrome-stained images as well as immunofluorescence images of arterial sections. Movat images were scored for VSMC loss and accumulation of proteoglycan. Immunofluorescence images were stained with DAPI, and smooth muscle $\alpha$-actin, a marker of differentiated vascular smooth muscle cells. Cellularity as measured by DAPIpositive nuclei in the media and the amount of smooth muscle $\alpha$-actin staining were scored separately. All scoring was done on a scale from 0 to 5 with 0 indicating no pathology and 5 indicating severe pathology. Results for individual mice were plotted based upon the average pathology scores among the five observers, and compared with the percentage of non-farnesylated HDJ-2 that appeared on Western blots of liver protein extracts obtained at the time of dissection.

Statistical Analysis. To test whether inhibition of farnesylation led to reduced pathology in the transgenic mice, a linear model was fit to the data, with

1. Meredith MA, et al. (2008) Phenotype and course of Hutchinson-Gilford progeria syndrome. N Engl J Med 358:592-604.

2. Eriksson M, et al. (2003) Recurrent de novo point mutations in lamin A cause Hutchinson-Gilford progeria syndrome. Nature 423:293-298.

3. De Sandre-Giovannoli A, et al. (2003) Lamin a truncation in Hutchinson-Gilford progeria. Science 300:2055.

4. Capell BC, Collins FS (2006) Human laminopathies: nuclei gone genetically awry. Nat Rev Genet 7:940-952.

5. Dechat T, et al. (2007). Alterations in mitosis and cell cycle progression caused by a mutant lamin A known to accelerate human aging. Proc Natl Acad Sci USA 104:4955-4960.

6. Beck LA, Hosick TJ, Sinensky M (1990) Isoprenylation is required for the processing of the lamin A precursor. J Cell Biol 110:1489-1499.

7. Varga R, etal. (2006) Progressive vascular smooth muscle cell defects in a mouse model of Hutchinson-Gilford progeria syndrome. Proc Natl Acad Sci USA 103:3250-3255.

8. Capell BC, Collins FS, Nabel EG (2007) Mechanisms of cardiovascular disease in accelerated aging syndromes. Circ Res 101:13-26.

9. Stehbens WE, Delahunt B, Shozawa T, Gilbert-Barness E (2001) Smooth muscle cell depletion and collagen types in progeric arteries. Cardiovasc Pathol 10:133-136.

10. Lin F, Worman HJ (1993) Structural organization of the human gene encoding nuclear lamin A and nuclear lamin C. J Biol Chem 268:16321-16326.

11. Hennekes H, Nigg EA (1994) The role of isoprenylation in membrane attachment of nuclear lamins. A single point mutation prevents proteolytic cleavage of the lamin A precursor and confers membrane binding properties. J Cell Sci 107(Pt 4):10191029.

12. Goldman RD, et al. (2004) Accumulation of mutant lamin A causes progressive changes in nuclear architecture in Hutchinson-Gilford progeria syndrome. Proc Natl Acad Sci USA 101:8963-8968.

13. Scaffidi P, Misteli T (2005) Reversal of the cellular phenotype in the premature aging disease Hutchinson-Gilford progeria syndrome. Nat Med 11:440-445.

14. Cao K, Capell BC, Erdos MR, Djabali K, Collins FS (2007) A lamin A protein isoform overexpressed in Hutchinson-Gilford progeria syndrome interferes with mitosis in progeria and normal cells. Proc Natl Acad Sci USA 104:4949-4954.

15. Csoka $A B$, et al. (2004) Genome-scale expression profiling of Hutchinson-Gilford progeria syndrome reveals widespread transcriptional misregulation leading to mesodermal/mesenchymal defects and accelerated atherosclerosis. Aging Cell 3:235-243.

16. Capell BC, et al. (2005) Inhibiting farnesylation of progerin prevents the characteristic nuclear blebbing of Hutchinson-Gilford progeria syndrome. Proc Natl Acad Sci USA 102:12879-12884. either the treatment dose or the percentage of non-farnesylated HDJ-2 as the explanatory variable and a separate variance component for each of the five blinded observers. The separate variance components were included to allow for random effects of random variation and potentially differing precision among the five observers. For a dichotomous explanatory variable (i.e., treatment with $0 \mathrm{tg}$ or $450 \mathrm{tg}$ ), this model was equivalent to a one-way ANOVA with random effects for observers. For a continuous explanatory variable (i.e., treatment with $0 \mathrm{tg}, 150 \mathrm{tg}$, or $450 \mathrm{tg}$; or percentage of non-farnesylated HDJ-2), this model was equivalent to linear regression with random effects for observers. For the initial group of mice that began treatment at time of weaning, we also included an indicator for age at dissection ( $\leq 300 \mathrm{vs}$. $>345 \mathrm{~d}$ ) as a covariate, as the age at dissection varied between 9 and 12 months for this group of mice. Trend lines on scatter plots represent the pathology levels predicted by percentage of non-farnesylated HDJ-2 under the linear model. A one-sided $P$ value $<0.05$ was considered evidence of a significant association of either FTI treatment or percentage of non-farnesylated HDJ-2 with reduced pathology.

ACKNOWLEDGMENTS. We thank Johnson \& Johnson, in particular David End for providing the FTI tipifarnib (R115777, Zarnestra), and Clinton Grubbs of the University of Alabama at Birmingham for providing data as well as his advice and expertise on the oral administration of R115777. We also thank the National Heart, Lung, and Blood Institute Light Microscopy Core Facility for assistance with the confocal images, Darryl Leja for his assistance in preparing the figures, and Hal Dietz for his valuable input on this study.

17. Toth Jl, et al. (2005) Blocking protein farnesyltransferase improves nuclear shape in fibroblasts from humans with progeroid syndromes. Proc Natl Acad Sci USA 102:12873-12878.

18. Glynn MW, Glover TW (2005) Incomplete processing of mutant lamin A in HutchinsonGilford progeria leads to nuclear abnormalities, which are reversed by farnesyltransferase inhibition. Hum Mol Genet 14:2959-2969.

19. Mallampalli MP, Huyer G, Bendale P, Gelb MH, Michaelis S (2005) Inhibiting farnesylation reverses the nuclear morphology defect in a HeLa cell model for HutchinsonGilford progeria syndrome. Proc Natl Acad Sci USA 102:14416-14421.

20. Fong LG, et al. (2006) A protein farnesyltransferase inhibitor ameliorates disease in a mouse model of progeria. Science 311:1621-1623.

21. Yang SH, et al. (2006) A farnesyltransferase inhibitor improves disease phenotypes in mice with a Hutchinson-Gilford progeria syndrome mutation. J Clin Invest 116:21152121.

22. Basso AD, Kirschmeier P, Bishop WR (2006) Lipid posttranslational modifications. Farnesyl transferase inhibitors. J Lipid Res 47:15-31.

23. Adjei AA, Davis JN, Erlichman C, Svingen PA, Kaufmann SH (2000) Comparison of potential markers of farnesyltransferase inhibition. Clin Cancer Res 6:2318-2325.

24. Widemann BC, et al. (2006) Phase I trial and pharmacokinetic study of the farnesyltransferase inhibitor tipifarnib in children with refractory solid tumors or neurofibromatosis type I and plexiform neurofibromas. J Clin Oncol 24:507-516.

25. Scaffidi P, Misteli T (2008) Lamin A-dependent misregulation of adult stem cells associated with accelerated ageing. Nat Cell Biol 10:452-459.

26. Gordon LB, et al. (2007) Disease progression in Hutchinson-Gilford progeria syndrome: impact on growth and development. Pediatrics 120:824-833.

27. Kieran MW, Gordon L, Kleinman M (2007) New approaches to progeria. Pediatrics 120:834-841.

28. Varela l, et al. (2008) Combined treatment with statins and aminobisphosphonates extends longevity in a mouse model of human premature aging. Nat Med 14:767-772.

29. Demierre MF, Higgins PD, Gruber SB, Hawk E, Lippman SM (2005) Statins and cancer prevention. Nat Rev Cancer 5:930-942.

30. Sugita M, Sugita $H$, Kaneki M (2007) Farnesyltransferase inhibitor, manumycin a prevents atherosclerosis development and reduces oxidative stress in apolipoprotein E-deficient mice. Arterioscler Thromb Vasc Biol 27:1390-1395.

31. Scaffidi P, Misteli T (2006) Lamin A-dependent nuclear defects in human aging. Science 312:1059-1063.

32. McClintock D, et al. (2007) The mutant form of lamin A that causes Hutchinson-Gilford progeria is a biomarker of cellular aging in human skin. PLOS ONE 2:e1269. 